\title{
On the general theory of the origins of retroviruses
}

Misaki Wayengera

\author{
Correspondence: wmisaki@yahoo. \\ com \\ Unit of Theoretical Biology, Division \\ of Molecular Pathology, \\ Department of Pathology, School \\ of Biomedical Sciences, College of \\ Health Sciences, Makerere \\ University, PO Box 7072, Kampala, \\ Uganda
}

\begin{abstract}
Background: The order retroviridae comprises viruses based on ribonucleic acids (RNA). Some, such as HIV and HTLV, are human pathogens. Newly emerged human retroviruses have zoonotic origins. As far as has been established, both repeated infections (themselves possibly responsible for the evolution of viral mutations ( $\mathbf{V m})$

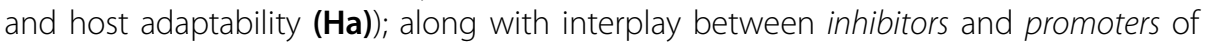
cell tropism, are needed to effect retroviral cross-species transmissions. However, the exact modus operadi of intertwine between these factors at molecular level remains to be established. Knowledge of such intertwine could lead to a better understanding of retrovirology and possibly other infectious processes. This study was conducted to derive the mathematical equation of a general theory of the origins of retroviruses.
\end{abstract}

Methods and results: On the basis of an arbitrarily non-Euclidian geometrical "thought experiment" involving the cross-species transmission of simian foamy virus (sfv) from a non-primate species Xy to Homo sapiens ( $\mathrm{Hs}$ ), initially excluding all social factors, the following was derived. At the port of exit from $X y$ (where the species barrier, SB, is defined by the Index of Origin, IO), sfv shedding is (1) enhanced by two transmitting tensors (Tt), (i) virus-specific immunity (VSI) and (ii) evolutionary defenses such as APOBEC, RNA interference pathways, and (when present) expedited therapeutics (denoted $\mathrm{e}^{2} \mathrm{D}$ ); and (2) opposed by the five accepting scalars (At): (a) genomic integration hot spots, glHS, (b) nuclear envelope transit (NMt) vectors, (c) virus-specific cellular biochemistry, VSCB, (d) virus-specific cellular receptor repertoire, VSCR, and (e) pH-mediated cell membrane transit, ( $\downarrow_{\mathrm{pH}}$ CMat). Assuming $\mathbf{A s}$ and $\mathbf{T t}$ to be independent variables, $\mathbf{I O}=\mathbf{T t} / \mathbf{A s}$. The same forces acting in an opposing manner determine SB at the port of sfv entry (defined here by the Index of Entry, $\mathbf{I E}=\mathbf{A s} / \mathbf{T t})$. Overall, If sfv encounters no unforeseen effects on transit between $X y$ and $H s$, then the square root of the combined index of sfv transmissibility $(\sqrt{ }|\mathbf{R T I}|)$ is proportional to the product IO* $\mathrm{E}$ ( $\mathrm{or} \sim \mathrm{Vm}^{*} \mathrm{Ha}^{*} \sum \mathrm{Tt}^{*} \sum A s^{*} \boldsymbol{\Omega}$ ), where $\boldsymbol{\Omega}$ is the retrovirological constant and $\Sigma$ is a function of the ratio Tt/As or As/Tt for sfv transmission from Xy to Hs.

Conclusions: I present a mathematical formalism encapsulating the general theory of the origins of retroviruses. It summarizes the choreography for the intertwined interplay of factors influencing the probability of retroviral cross-species transmission:

$\mathrm{Vm}, \mathrm{Ha}, \mathrm{Tt}, \mathrm{As}$, and $\Omega$. 


\section{Background}

The order Retroviridae constitutes a collection of non-icosahedral, enveloped viruses with two copies of a single-stranded RNA genome [1-5]. Retroviruses are known to infect avians [1] and murine [2], non-primate [3] and primate [4,5] mammals. Viruses of the order Retroviridae are unique in the sense that they can reverse-transcribe their RNA into complementary DNA, which is eventually integrated into the host genome (see Figure 1 for illustration of HIV replicative cycle) [6]. This intermediate DNA phase between RNAs may make retroviruses a valuable model for developing general virological concepts.

Two human retroviruses of the family Lentiviridae are known, Human Immunodeficiency Virus (HIV, which causes AIDS) $[5,6]$ and Human T cell Leukamia Virus




(HTLV a causative agent of leukemia) [4]. Emerging human retroviruses, previously undocumented in man, appear to arise by zoonotic transmission. For example, there is evidence that HIV emerged in humans after multiple independent zoonotic events involving cross-species transmissions of simian immunodeficiency viruses (SIVs) from nonhuman primates [5]. SIVs are phylogenetically very close to HIV, corroborating the role of SIV mutation $(\mathrm{Vm})$ or recombination in the origin of HIV [7]. Similar crossspecies transmission of retroviruses, though rarely observed among lower mammals, has been reported between felines and pumas $[8,9]$. These rare incidences seem to be preceded by a repeated assault (or 'attempt') on the host by the retrovirus. For example, in a recent investigation of feline immunodeficiency virus infection among bobcats and pumas in Southern California, Franklin et al. [8] provide evidence that cross-species infections have occurred frequently among these animals leading to the eventual transmission of the virus (FIV) to puma. The above data imply the existence of a biological restriction on cross-species retroviral transmissions, the species barrier (SB) [8]. For the purposes of this work, SB was defined as a biological barricade that inherently restricts cross-species transmission of retroviruses but, when jumped, enables such transmission. The repeated host assaults needed by the retrovirus to achieve cross-species transmission may also suggest that a level of host adaptation (as well as retroviral mutation or recombination) is required to effect the SB jump. This is consistent with the postulates of an earlier hypothesis I advanced to explain origins of retroviruses $[10,11]$.

It is well established that repeated contact between a potential new and a known reservoir host plays a role in breaching the $\mathrm{SB}$, but the dynamics of the underlying molecular mechanisms remain ill-defined. Current understanding may suggest that a threshold of retroviral load is needed to achieve inoculation, or viral mutation $(\mathrm{Vm})$ and possibly new host adaptation $(\mathrm{Hm})$ is needed to achieve retroviral cross-species transmission [8-12]. All in all, recent evidence for the regular transmission of primate retroviruses suggests that zoonosis, per se, may not be the rate-limiting step in pandemic retrovirus emergence, and that other factors such as viral adaptation are probably important for successful crossspecies transmission and a human pandemic [12]. Vandewoude et al. [9] used an experimental model to establish that although domestic cats (Felis catus) are susceptible to FIVs originating from pumas or lions, the circulating virus is reduced to nearly undetectable levels in most animals within a relatively short time. This diminution of viral load was found to be proportional to the initial viral peak, suggesting that the non-adapted host successfully inhibits normal viral replication, leading to replication-incompetent viral progeny. The possible mechanisms proposed for such restriction of cross-species infection in natural settings include: (1) lack of conducive contact between infected and shedding animals of different species; (2) lack of a suitable receptor repertoire to allow viral entry into susceptible cells of the new species; (3) a sufficient difference in cellular machinery between the new and the primary host to preclude viral replication; (4) intracellular restriction mechanisms in the new host that limit viral replication; (5) ability of the new host to raise sterilizing adaptive immunity, resulting in aborted infection and inability to spread infection among con-specifics; or (6) production of defective or non-infectious viral progeny that lack the cellular cofactors required to infect conspecifics [5]. Overall, these data support the view that there is a unique requirement for retroviral fitness $(\mathrm{Vm})$ and for host adaptability (Ha) to effect the SB jump. The same work also points to the 
existence of intracellular restriction mechanisms for cross-species retrovirus transmission (hereafter denoted transmitting tensors, $\mathbf{T t}$ ) as well as intracellular mechanisms that can promote inter-species transmission of retroviruses (hereafter denoted accepting scalars, As) $[8,9,12]$.

The purpose of this work was to derive a mathematical formalism that integrates and expresses the molecular interplay among $\mathrm{Vm}, \mathrm{Ha}, \mathrm{Tt}$ and As during enhancement or breach of the SB when retroviruses are transmitted across species. On the basis of an arbitrarily non-Euclidian geometrical "thought experiment" involving the cross-species transmission of simian foamy virus (sfv) from a non-primate species $X y$ to Homo sapiens $(H s)$, initially excluding all social factors, the following was derived. At the port of sfv exit from Xy (where SB is defined by the Index of Origin-IO); sfv shedding is (1) enhanced by the two tensors (Tt): (i) virus specific immunity (VSI) [13-15] and (ii) evolutionary defenses such as APOBEC [16-19], Tripartite Motif (TRIM) family [20], interferon-induced transmembrane protein BST-2 (CD317; tetherin) [21], RNA interference pathways [22-24], plus, where present, expedited therapeutics (all denoted $\mathrm{e}^{2} \mathrm{D}$ ); and (2) opposed by the five Accepting scalars (As): (a) genomic integration hot spots-gIHS [25-33], (b) nuclear membrane transit (NMt) vectors[6], (c) virus specific cellular biochemistry-VSCB[6], (d) virus specific cellular receptor repertoire-VSCR [34-39], and (e) pH mediated cell membrane transit-( $\downarrow_{\mathrm{pH}}$ CMat) [40-42]. The scalar function, as used here in biological space-time, differs from its physical analogue in that it exhibits both magnitude and direction (in contrast to physics, where scalars only have magnitude) that are equal and opposite to the tensor function. Assuming As and $\mathbf{T t}$ to be independent variables, $\mathbf{I O}=\mathbf{T t} / \mathbf{A s}$. The same forces acting in an opposing manner determine SB at the port of sfv entry (defined here by the Index of Entry, $\mathbf{I E}=\mathbf{A s} / \mathbf{T t}$ ). Overall, if sfv encounters no unforeseen effects on transit between $X y$ and $H s$, the square root of the combined index of sfv transmissibility $(\sqrt{ }|\mathbf{R T I}|)$ is proportional to the product IO* IE (or $\sim \mathrm{Vm}^{*} \mathrm{Ha}^{*} \sum \mathrm{Tt}^{* *} \Sigma \mathrm{As} s^{*} \Omega$ ); where $\Omega$ is the retrovirological constant, and $\sum$ is a function of the ratios $\mathrm{Tt} / \mathrm{As}$ or As/Tt for this particular arbitrary event of sfv transmission from $\mathrm{X} y$ to $\mathrm{H} s$.

\section{Methods and approach}

\section{The "thought experiment"}

First, to contemplate the mathematical scope of the dynamics of retroviral cross-species transmission, I concocted a thought experiment involving the transmission of a retrovirus-simian foamy virus ( $\mathrm{sfv}$ ) from the arbitrary non-human primate species $X y$ to Homo sapiens. The system was imagined to exclude all social factors such as contact and contact repetition; it was assumed that only biological factors influence retroviral cross-species transmissions, until another constant is introduced that may also integrate social factors, the retrovirological constant. In this "thought experiment", sfv must first break free from the influence of the net of molecular determinants of SB in $X y$ (the component of SB here being derived as the Index of Origin, IO) before entering $H s$ by similarly overcoming the SB determinants there (the relevant component of SB being defined by the Index of Entry, IE).

In order to derive the pathway of sfv mathematically, I observed that only the kind of non-Euclidian geometry that represents curvature in space-time may suffice. This led me to recruit an unlikely-seeming comparison between physical and biological 
phenomena (unlikely since the former are mostly concerned with constants while the latter largely involve dynamic processes that differ among species and individuals). Specifically, I re-envisaged the dynamics of sfv cross-species transmission as analogous to those of a comet traveling from Mars to earth. Such a comet must first break through the gravitational and atmospheric fields of Mars (analogous to the point when sfv breaks free of the net effect of IO operating in $X y$ ) and then move through free space until it breaks through the earth's atmospheric and gravitational fields (analogous to the point at which sfv breaks through the IE in $H s$ ) (see figure 2). The path of such a comet is best described by Einstein's field equation of gravitation $\left(\mathbf{R}_{\mu v}-\mathbf{1} / \mathbf{2} \mathbf{g}_{\mu v} \mathbf{R}=\mathbf{8} \mathbf{T}_{\mu v}\right.$, where $\mathbf{R}_{\boldsymbol{\mu}}$ is the Ricci Tensor, $\mathbf{g}_{\mu \nu}$ is the metric tensor, $\mathbf{R}$ is the Ricci scalar, and $\mathrm{T}$ is the all-important Einstein's tensor) [43-45]. The dynamics of retroviral cross-species transmissions do not really resemble such physical phenomena, but this arbitrary comparison crucially led to the insight that non-Euclidian tensors may similarly be used to represent the SB variables $\mathbf{V m}, \mathbf{H a}, \mathbf{T t}$ and As at the ports of both sfv origin and exit [46].

Tensors are vectors that contain multiple independent variables possessing both direction and magnitude. In Euclidian geometry, increases in the number of components account for various dimensions of visualization. For instance, in 2-D, every tensor has three components; six components are integral in a 3-D tensor, and 10 in a tensor of 4-D (the realm of physical space-time) [46]. The non-Euclidian space-time tensors that Einstein used to derive his field equations of gravitation have over 16 independent components [43-45]. Thus, to assume that cross-species retroviral
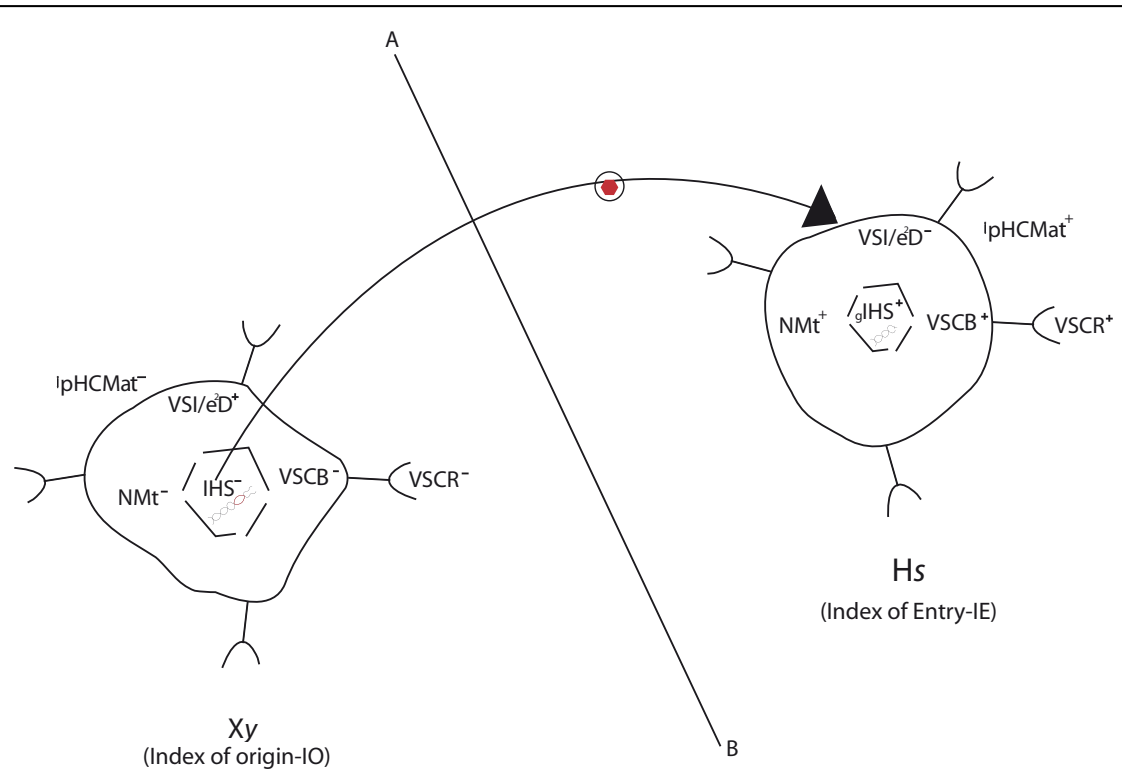

$\mathrm{Hs}$

(Index of Entry-IE)

Figure 2 Schematics of the imagined trajectory of a virus (sfv) jumping from one species $(X y)$ to another $(H s)$. The figure is based on the assumption that a retrovirus experiences: (1) an $X y$-component of the SB denoted the Index of Origin or IO; (2) at $\mathrm{Hs}$ the index of entry or IE. The path of such a retrovirus is analogous to the trajectory of an object cast from one planet's gravitational and atmospheric field into another's. The path of such a physical phenomenon is described by Einstein's field equations of gravitation $\left(\mathbf{R}_{\mu \boldsymbol{v}}-\mathbf{1 / 2} \mathbf{g}_{\boldsymbol{\mu \nu}} \mathbf{R}=\mathbf{8} \mathbf{T}_{\boldsymbol{\mu \nu}}\right.$, where $\mathbf{R}_{\boldsymbol{\mu}}$ is the Ricci Tensor, $\mathbf{g}_{\boldsymbol{\mu} \boldsymbol{v}}$ is the metric tensor, $\mathbf{R}$ is the Ricci scalar, and T is the all important Einstein's tensor) [43]. Analogously, at the port of sfv exit from Xy (where SB is defined by the $(0)$, sfv shedding is (1) enhanced by the two transmitting tensors (Tt) and (2) opposed by the five accepting scalars (At), as described in the text. The same forces acting in an opposing manner determine $S B$ at the port of sfv entry (defined here by the IE). 
transmission assumes a path closely similar to that of the physical phenomena has implications for the nature of the variables Vm, Ha, Tt and As: (i) Vm, Ha, Tt and As are non-Euclidian tensors in 4-D comprising 16 or more components; (2) they are covariant in nature, meaning that there can only be one possible finite value for each. The influence on SB jump dynamics of a change in the finite value of any of the 16-plus components is balanced by reciprocal changes in the others, ensuring the constancy of $\mathbf{V m}, \mathbf{H a}, \mathbf{T t}$ and As. The unique advantage of this approach is that only a few of the components need to be known for a mathematical formalism of the theory of retroviral transmission to be obtained. This is important because not all the molecular determinants of retrovirus species cross-species transmission are known.

\section{Annotation of the non-Euclidian biological tensors/scalars $\mathrm{Vm}, \mathrm{Ha}, \mathrm{Tt}$ and As}

Second, to annotate the components of the non-Euclidian tensors and scalars operating in this imagined scenario of sfv cross-species transmission (the full composition may remain uncertain because many determinants are still poorly understood), I followed sfv on its imagined path through each compartment of $X y$ and $H s$, defining and positioning the currently-known biological determinants of the transmission process (see Figure 2). At the port of sfv exit from $X y$ (defined by IO), sfv shedding is (1) enhanced by the two transmitting tensors (Tt) and (2) opposed by the five accepting scalars (At) explained above. Continuing the thought experiment, the same factors are bound to operate at the port of sfv entry into $H s$ (synonymous with IE), except that what were annotated as transmitting tensors become accepting scalars, and vice versa. Because each individual tensor and scalar was annotated to be largely compartmentalized, it seemed appropriate to consider rules of multiplication or fractionation to govern their future combinations, since mathematically they may be considered mutually independent. Hence, assuming As and Tt within the same host to be independent variables, then $\mathbf{I O}=\mathbf{T t} / \mathbf{A s}$. (When similar forces act in an opposing manner to determine SB at the port of sfv entry, IE = As/Tt).

Overall, two major assumptions were made throughout these derivations. First, only biological factors were considered, leaving social factors such as contact and contact repetition aside; several existing models deal with those [47-53], and a subsequently introduced covariant, the retrovirological constant, may be used to account for them. Second, I assumed that the retrovirus sfv experiences no uncertain influences of any mode or origin between its ports of exit and entry [46]. This is obviously a major presumption, especially since most effective "public health control measures" would best be situated between those ports.

What are arbitrarily annotated as tensors and scalars represent, in real biology, innate or acquired ecological responses of the retrovirus/host to variations in population-wide dynamics, and some may be subject to adaptation. The resulting unpredictable behavior of biological systems, in contrast to physical phenomena, underlines the fact that possibly no single physico-mathematical system can portray events in biology sustainably over time, unless it (a) leaves open a window to allow for uncertainty arising from biological unpredictability, and (b) recognizes retroviral transmission as analogous to a dual wave-particle phenomenon. This view led to the concept of a retrovirological window (discussed below) and use of a mosaic of quantum and relativistic approaches [54] to define qualitatively the range of space-time in the retrovirological fields over which the equations advanced may be accurate (see Figure 3). 


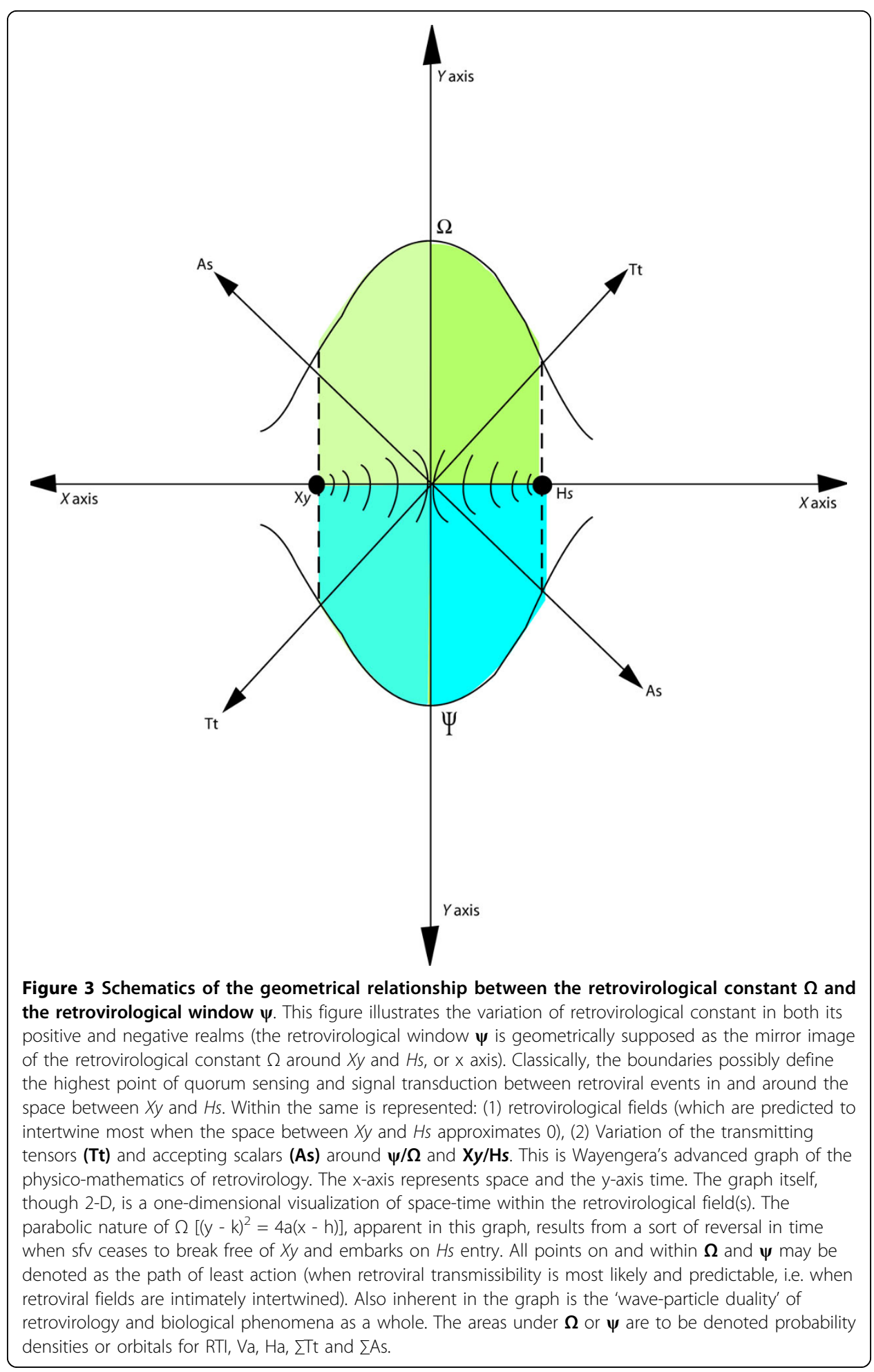




\section{Derivation of the equivalent of $\mathrm{IO}$ and/or IE}

From the arbitrary annotation of forces influencing sfv $X y$ exit or $H s$ entry, it may be stated mathematically that:

$$
\begin{aligned}
& \text { IO } \propto \text { Transmitting Tensors / Accepting } \\
& \text { Scalars } \\
& \text { IE } \propto \text { Accepting Scalars / Transmitting } \\
& \text { Tensors }
\end{aligned}
$$

However, As may currently be represented as proportional to:

$$
\begin{aligned}
& \text { As } \propto \text { VSCR }^{*} \downarrow_{\mathrm{pH}} \\
& \text { CMat }{ }^{*} \text { VSCB }{ }^{*} \text { NMt }{ }^{*} \text { gIHS }
\end{aligned}
$$

And Tt may mathematically be denoted as follows:

$$
\begin{aligned}
& \text { Tt } \propto \\
& \text { VSI }{ }^{*} e^{2} D
\end{aligned}
$$

From equations iii and iv, equations $\mathrm{i}$ and ii become re-expressible as $\mathrm{v}$ and vi below, respectively. Two further major assumptions are made here to remove the proportionality sign and replace it with an equal sign.

- In the first instance, it was necessary to introduce within the transmitting tensors an arbitrary constant of innate or acquired viral fitness specific to the retrovirus concerned, denoted $\lambda$. At the ports of exit and entry, retroviral fitness is denoted respectively $\lambda^{\mathbf{0}}$ and $\lambda^{\prime}$. These factors serve to illustrate that, even if viral mutation (or phenotypic adaptability) is already noted as a major player in retroviral cross-species transmissibility, it is tailored to the retrovirus in question; some retroviruses are predictably more mutable than others. Also, because the several non-Euclidean components of each transmitting tensor (compartments VSI and $\mathrm{e}^{2} \mathrm{D}$ used here) remain illdefined, arbitrary multiplying factors were introduced for each transmitting tensor compartment, $\pi 1$ and $\pi 2$ for VSI and $e^{2} D$ respectively; their integral products are $\pi^{\mathbf{0}}$ or $\pi$ ' within the host of origin and that of entry respectively.

- On the other hand, for the accepting scalars, a constant for specific host adaptability $(\phi)$ was necessary to formalize the dynamics of retroviral cross species transmissibility correctly and comprehensively; $\phi^{\mathbf{0}}$ and $\phi^{\prime}$ for $X y$ and $H s$ respectively. In addition, as for the tensors, the relationships among the five independent accepting scalar compartments listed should have a governing proportionate factor for each (since their full composition is apparently unknown): $\kappa 1, \kappa 2, \kappa 3, \kappa 4$ and $\kappa 5$ respectively for VSCR, $\downarrow_{\mathbf{p H}} \mathbf{C M a v t ,}$ VSCB, NMt and gIHS; the derivative products are $\kappa^{\mathbf{0}}$ and $\kappa^{\prime}$.

Hence,

$$
\begin{aligned}
& \mathrm{IO} \propto\left[\mathrm{VSI}{ }^{*} \mathrm{e}^{2} \mathrm{D}\right]^{*} 1 /\left(\mathrm{VSCR}^{*} \downarrow_{\mathrm{pH}}\right. \\
& \left.\mathrm{CMat}^{*} \mathrm{VSCB}^{*} \mathrm{NMt}{ }^{*} \mathrm{gIHS}\right)
\end{aligned}
$$


may be approximated to:

$$
\begin{aligned}
\mathrm{IO} & =\pi^{0 *} \boldsymbol{\varphi}^{0} *\left[\mathrm{VSI}{ }^{*} \mathrm{e}^{2} \mathrm{D}\right]^{0 *} 1 / \\
& {\left[\boldsymbol{\kappa}^{0} * \lambda^{0 *}\left(\mathrm{VSCR}^{*} \downarrow_{\mathrm{pH}} \mathrm{CMat}^{*} \mathrm{VSCB}^{*} \mathrm{NMt}^{*} \mathrm{gIHS}\right)\right]^{0} }
\end{aligned}
$$

Similarly,

$$
\begin{aligned}
& \text { IE } \propto\left[\text { VSCR }^{*} \downarrow_{\mathrm{pH}} \mathrm{CMat}^{*} \mathrm{VSCB}^{*} \mathrm{NMt}^{*} \mathrm{gIHS}^{*} \mathbf{1} /\right. \\
& \left(\mathrm{VSI} * \mathrm{e}^{2} \mathrm{D}\right)
\end{aligned}
$$

may be approximated to:

$$
\begin{aligned}
\mathrm{IE}= & \kappa^{\prime *} \lambda^{\prime *}\left[\mathrm{VSCR}^{*} \downarrow_{\mathrm{pH}} \mathrm{CMat}^{*} \mathrm{VSCB}^{*} \mathrm{NMt}^{*} \mathrm{gIHS}\right]^{\prime *} 1 / \\
& \left\{\boldsymbol{\pi}^{\prime *} \varphi^{\prime *}\left[\left(\mathrm{VSI}^{*} \mathrm{e}^{2} \mathrm{D}\right)^{\prime}\right\}\right.
\end{aligned}
$$

Formulation of the integral equation: the relative transmissibility index (RTI)

From equations v.b and vi.b, the relative transmissibility index (RTI) may be mathematically formalized as:

$$
\begin{aligned}
& \mid \text { RTI } \mid \propto \\
& \text { IO * IE }
\end{aligned}
$$

Substituting from equations v.b and vi.b:

$$
\begin{aligned}
& \mid \text { RTI } \mid \propto \boldsymbol{\pi}^{0} * \boldsymbol{\varphi}^{0} *\left[\mathrm{VSI} * \mathbf{e}^{2} \mathrm{D}\right]^{0 *} \boldsymbol{\kappa}^{\prime *} \boldsymbol{\lambda}^{\prime *} \\
& \left(\text { VSCR }^{*} \downarrow_{\mathrm{pH}} \mathrm{CMat}^{*} \text { VSCB }^{*} \mathrm{NMt}^{*}\right. \text { gIHS) } \\
& { }^{*} \boldsymbol{\pi}^{\prime *} \boldsymbol{\varphi}^{\prime *}\left[\mathrm{VSI} * \mathrm{e}^{2} \mathrm{D}\right]^{\prime *} \boldsymbol{\kappa}^{0} * \boldsymbol{\lambda}^{0} * \\
& \left(\text { VSCR }^{*} \downarrow_{\mathrm{pH}} \mathrm{CMat}^{*} \mathrm{VSCB}^{*} \mathrm{NMt}^{*} \text { gIHS }\right)^{0} \\
& \mid \text { RTI } \mid \propto \lambda^{\prime *} \varphi^{0 *} \pi^{0}\left[\text { VSI } * \mathbf{e}^{2} \mathrm{D}\right]^{0 *} \boldsymbol{\kappa}^{\prime} \\
& \text { (VSCR }^{*} \downarrow_{\mathrm{pH}} \mathrm{CMat}^{*} \mathrm{VSCB}^{*} \mathrm{NMt}^{*} \text { gIHS) } \\
& \lambda^{0} * \boldsymbol{\varphi}^{\prime *} \boldsymbol{\pi}^{\prime}\left[\mathrm{VSI}{ }^{*} \mathrm{e}^{2} \mathrm{D}\right]^{* *} \boldsymbol{\kappa}^{0} \\
& \left(\text { VSCR }^{*} \downarrow_{\mathrm{pH}} \mathrm{CMat}^{*} \text { VSCB }^{*} \mathrm{NMt}^{*} \text { gIHS }\right)^{0}
\end{aligned}
$$

Further major simplifications may now be introduced:-

- First, $\lambda^{\prime} / \lambda^{\mathbf{0}}$ may be considered equivalent to specific viral mutability: $\mathbf{V m}$

- Second, $\phi^{\mathbf{0}} / \phi^{\prime}$ is the inverse of host mutability, termed host adaptability: Ha

- Third, the complex factor $\boldsymbol{\pi}^{\mathbf{0}}\left[\mathbf{V S I} \mathbf{I}^{2} \mathbf{D}\right]^{\mathbf{0}_{*}} \mathbf{1} /\left(\boldsymbol{\pi}^{\prime}\left[\mathbf{V S I}^{*} \mathbf{e}^{2} \mathbf{D}\right]^{\prime}\right)$ equals the effective Net Transmitting tensor: $\Sigma \mathbf{T t}$

- Forth, the complex factor $\kappa^{\prime}\left(\text { VSCR }^{*} \downarrow_{\mathbf{p H}} \text { CMat }^{*} \text { VSCB }^{*} \text { NMt }^{*} \text { GIHS }\right)^{* * 1} 1 /\left[\kappa^{0}\right.$ (VSCR $\left.\left.{ }^{*}{ }_{\mathbf{p H}} \mathbf{C M a t}^{*} \mathrm{VSCB}^{*} \mathbf{N M t}^{*} \mathbf{g I H S}\right)^{\mathbf{0}}\right]$ represents the effective net accepting scalar: $\Sigma$ As

As used here, $\Sigma$ denotes a function of the ratio $\mathrm{Tt} / \mathrm{As}$ for sfv transmission from $X y$ to $H s$, and not its usual formal mathematical implication of summing. 
Hence,

$$
|\mathrm{RTI}| \propto \mathrm{Vm}^{*} \mathrm{Ha} * \Sigma \mathrm{Tt} * \Sigma \mathrm{As}
$$

In order to replace the proportionality sign with an equal sign, a new constant, the retrovirological constant $(\Omega)$, is introduced. This brings us to the final equation advanced for the general theory of retrovirology:

$$
|\mathbf{R T I}|=\mathrm{Vm}^{*} \mathrm{Ha}^{*} \Sigma \mathrm{Tt}^{*} \Sigma \mathrm{As}^{*} \boldsymbol{\Omega}
$$

Observe that, if one alternatively purposed to consider Tt and As operating within the same host as dependent variables (a scenario I disregarded since it makes the biological phenomenon nearly homologous to physical phenomena), then, by maintaining $\mathrm{Vm}$ and $\mathrm{Ha}$ as independent, the same equation ix may be re-phrased as: $|\mathbf{R T I}|=\Sigma(\mathrm{Tt}$ As) $)_{\mathrm{Xy}}{ }^{*} \Sigma(\mathrm{As}-\mathrm{Tt})_{\mathrm{Hs}}{ }^{*} \mathrm{Vm} * \mathrm{Ha} * \boldsymbol{\Omega}$; in which case $\Sigma$ retains its mathematical meaning of summing.

Is this just another mathematical attempt at biology, or it is something that may add to our knowledge of retrovirology and possibly other infectious pathogen transmission dynamics? It is an enormous and serious challenge to simplify and unify retrovirology. I discuss below the ramifications I have so far seen of the proposed formalism; readers may find other insights. In addition, I suggest experiments that may be undertaken to test how well this equation represents retroviral cross-species transmission dynamics.

Additional modifications are made to the formalism as I re-visualize it in the light of the existing literature in physics, mathematics and retrovirology.

\section{Discussion}

The mathematical formalism of the theory of the origins of retroviruses presented above suggests that retroviral cross-species transmission results from a random yet geometrically predictable intertwining of $\mathrm{Vm}, \mathrm{Ha}, \mathrm{Tt}, \mathrm{As}$, and $\Omega$, a pattern consistent with the four postulates of the evolutionary adaptation cross-species (EACS) hypothesis I previously advanced to explain the origin of human viruses, the scope of which I have since limited to retroviruses.

First $\left(\mathrm{P}_{1}\right)$, emerging and re-emerging retroviruses exist before they are isolated or there is evidence that they cause human disease. They existed in previous hosts called "reservoirs", mostly wild game species, on which they depended for the virus-host cell interaction necessary for survival - making all retroviruses zoonotic in origin.

Second $\left(\mathrm{P}_{2}\right)$, with an increased change in variables among the reservoirs and chance of contact with a new host (humans), these retroviruses adapted, possibly but not necessarily through mutation, recombination and re-assortment to yield new strains with better fitness to use human cells for replication.

Third $\left(\mathrm{P}_{3}\right)$, for all newly emerging retroviruses, the most susceptible new hosts are those whose cellular biochemistry and genetics favors establishment of the virus by coding for and producing the necessary energy, metabolites and most (or in some cases all) the enzymes required for replication of the adapted new strain. Depending on the endogenous tissue specificity (fitness) exhibited by a retrovirus; however, retroviral cross-species jumps are possible between host species of variable biochemical and genetic homology. 
Fourth $\left(\mathrm{P}_{4}\right)$, various mechanisms of interaction between the previous and new (human) hosts are required to effect cross-species retroviral transmission. The mechanisms of transfer may be either direct (e.g. via human consumption of NHP game meat for simian immunodeficiency viruses such as SFV, or transplantation with porcine tissue for PERV-xenosis) or indirect, by vector transfer (a predicted scenario that may occur, say, with retrovirus-based bio-weaponry, discussed below) $[10,11]$. The general equation derived above suggests potentially interesting though not yet fully comprehensive ideas on: (1) the possible ramifications of this physicomathematical formalism of retrovirology and (2) the experiments that may be needed to test it.

\section{Ramifications of the equation of the theory of retrovirology}

\section{Insights into the overall dynamics of cross-species transmission}

From the final equation of retrovirus origin, the imaginary scenario involving transmission of the simian foamy virus (SFV) from the non-human primate species $X y$ to $H s$ may be considered as follows: whenever the net biological Tt and As within the animal host $X y$ is greater (in favor of Tt within $X y$ ) than the corresponding value in $H s$, there is a greater probability of cross-species transmission (best visualized using the variant of the equation that assumes $\mathrm{Tt}$ and As operating within the same host to be dependent variables, $\left.|\mathbf{R T I}|=\Sigma(\mathrm{Tt}-\mathrm{As})_{\mathrm{Xy}}{ }^{*} \boldsymbol{\Sigma}(\mathrm{As}-\mathrm{Tt})_{\mathrm{Hs}}{ }^{*} \mathrm{Vm}^{*} \mathrm{Ha}^{*} \boldsymbol{\Omega}\right)$. Conversely, greater net $\mathrm{Tt}$ and As in $H s$ than in $X y$ (in favor of $\mathrm{Tt}$ within $H s$ ) will disfavor sfv transmission. Thus, intense retrovirus (sfv)-specific immune responses in the animal host will enhance retroviral shedding and hence cross-species transmission, while similar responses in $H s$ will restrict viral tropism there. In general, any change among the covariants $\mathbf{V m}, \mathrm{Ha}, \mathbf{T t}$, As and $\boldsymbol{\Omega}$ that makes the $|\mathbf{R T I}|>1$ will favor the specified direction of cross-over of the arbitrary retrovirus sfv (from $X y$ to $H s$ ), while co-variations making $|\mathbf{R T I}|<1$ will disfavor it.

Wherever there is VSI (annotated as Tt), the natural reservoir elicits no retrovirusspecific immune responses (and VSI is possibly always zero or one). However, within the same natural reservoir setting, where retroviruses live harmoniously with the host, the equation predicts that artificial stimulation of virus-specific immune responses will favor viral shedding. Whether interactions between $\mathrm{X} y$ and $\mathrm{H} s$ can elicit VSI within $\mathrm{X} y$ remains to be established, but this would make retroviral shedding an adaptive response mounted by $\mathrm{X} y$ to protect its niche from encroachment by $\mathrm{Hs}$, and the retroviruses themselves would be commensals with guardian characteristics. This implies that vaccination of the reservoir host, unless it entirely eliminates the retrovirus, cannot reduce the risk of retroviral cross-species transmission and may indeed enhance it. Because the natural reservoir elicits no immune responses to the pathogen, this may explain the difficulties and discordance of results obtained by filovirus-specific IgG/M antibody detection tests and virus capture assays during the search for a natural reservoir of the re-emerging filoviruses Ebola and Marburg. Applying the field equations of retrovirology, it can be predicted that no virus will be detected within the natural reservoir, even when the filovirus is present, because there are no filovirus-specific immune responses. This renders assays of pathogen immune responses within the host inappropriate for studies that aim to identify the natural hosts of any pathogen (and techniques for the isolation of the pathogen Koch's style must continue to be 
considered). The equation of retrovirology also suggests that the 'purpose' of repeated zoonotic transmission of a retrovirus, such as that involving various SIV isolates reported between non-human primates and humans, is as follows. (a) It fine-tunes the new host's adaptability to sustain actively facilitated retroviral replication including (i) the selective adaptation of a permissive receptor repertoire where absent, (ii) recruitment of alternative biochemical pathways, and (iii) development of mechanisms for inhibition or outright evasion of any inherent inhibitory mechanisms within the target host such as APOBEC [16-19], interferon-induced transmembrane protein BST-2 (CD317; tetherin) [21], TRIM [20] and RNAi [22-24], present within most mammals. (b) It facilitates retroviral mutation or recombination. These two teleological reasons for repeated retroviral infections of a new host before the ultimate jump of the SB allow for the evolution of (1) host adaptations and (2) viral mutations or recombinations that will interact to make the host and virus fitted to cohabit $[12,47]$.

This implies that a "bio-weapon" may be developed in the laboratory by continuous cycles in which an acutely fatal retrovirus of zoonotic origin is co-cultured in human cell lines, rendering the human cells permissive to that retrovirus tropism. The same procedure may be used to select an appropriate animal carrier or "vector", say chickens, pigs, or even cows. Herein, shedding of the retroviral bio-agent may be enhanced by vaccination of these vector-hosts if they are appropriately adapted to act as natural hosts. Several other models for retrovirus-based bio-weaponry are possible, including starting with a known human retrovirus such as HIV and recombinantly engineering it to be acutely fatal (say by pseudo-enveloping it with or enabling it to express Ebola/ Marburg gp1, 2, the major pathogenic protein of filovirus hemorrhagic fever). Additional modifications such as altering the transmission dynamics of the retrovirus from contact with infected body fluid to air- or water-borne transmission would make it more damaging, though it is not immediately clear how that could be achieved.

More peacefully and productively, the mathematical formalism of retrovirology advanced here also underscores strategies for avoiding or mitigating the impact of retrovirus-based bio-weapons, such as the development of therapeutic interventions and avoidance of contact (see below).

\section{Retrovirological fields and their action}

From the final equation of the general theory and the platform of "thought experimentation" that relates biological and physical phenomena, 'retrovirological fields' around hosts may be imagined, analogous to gravitational and atmospheric fields around planets. This proposal arises from, and in support of, the assumption that a retrovirus crossing from one host to another encounters two barriers. Although the covariant nature of the tensors $\mathbf{V m}, \mathrm{Ha}, \mathbf{T t}$ and As makes their overall finite value appropriately covariant for deriving retrovirological fields mathematically, the real wave (or possibly quantum) pattern of such fields is best accounted for in the parabolically covariant nature of the retrovirological constant, as discussed below. In brief, the scenario is as follows: just as the mathematical formalism of retrovirology advanced here predicts that retroviruses within wild game (constantly adapting) are ever mutating (to expand their fields of retrovirological operation), so it is predictable that retrovirological fields will never be constant. This invites the question: "how may retroviruses themselves influence retrovirological fields, or vice versa?" From the equation of retrovirology, the 
central dogma of retrovirus transmissibility is: "Retroviral mutations serve to induce changes (by either approximation or distancing) within the retrovirological fields operating around hosts in a similar way to host adaptability". Hence, just as in the relationship between matter and space-time - "matter tells space-time how to curve, and curvature in space-time tells matter how to move" [43-45] - I believe that 'retroviruses and their hosts tell retrovirological fields how to change, and changes in retrovirological fields tell retroviruses and hosts how to mutate, adapt or transmit' [47].

Evidence in support of this interplay arises from data that link human activities such as hunting, mining, etc., which bring man into close contact with wild game harboring retroviruses such as HTLV-3/4, with human infection by the same [3,7]. In other words: the general theory of retrovirology provides the choreography for an intertwined dance of chance of retroviral cross-species transmission, $\mathbf{V m}, \mathbf{H a}, \mathbf{T t}, \mathbf{A s}$, and $\boldsymbol{\Omega}$ (see Figure 3 for illustration).

The retrovirological constant and its parabolically covariant nature: highest peak at the closest intertwining of retrovirological fields

Perhaps the greatest theoretical predicative power of the mathematical formalism of origins of retroviruses lies in its ability to elucidate the nature of a still-ambiguous constant, the retrovirological constant. Although the mathematical significance of the retrovirological constant in ensuring a balance between both sides of the equation is apparent, its finite value is not. Nevertheless, several predictions can be made about its nature and scope.

First, if the retrovirological constant is a non-Euclidean tensor as stated in equation ix, then, assuming that biological space-time is 4-D, it comprises 10 or more independent components [43-46]. Therefore, because the equation of retrovirology was derived purely on the basis of biological determinants of retroviral cross-species transmissions, the retrovirological constant may also be taken to incorporate several social determinants of retroviral transmissibility including mode of contact, contact repetitions [48-52] and the basic reproductive number of the retrovirus $\left(\mathrm{R}_{0}\right)$ [53]. In other words, the retrovirological constant is itself a non-Euclidian tensor that allows space for as many currently unknown factors in retrovirology as may be conceived. Alternatively, because the equation advanced says nothing about the virulence of sfv once it successfully integrates into the cells of the new host, $H s$, a virulence factor may appropriately be integrated into the constant.

Second, just as the tensors and scalars $\mathbf{V m}, \mathbf{H a}, \mathbf{T t}$ and As are predictably covariant, the retrovirological constant $\boldsymbol{\Omega}$ is similarly covariant. The covariance of $\boldsymbol{\Omega}$ implies that, as some of its components change, there is a tactically balanced adjustment in others so that at any given time its tensor value remains constant [43-46].

Third, since the probability of retroviral transmission increases as $X y$ and $H s$ become more closely intertwined, we have assigned a "parabolic" pattern to the influence of $\boldsymbol{\Omega}$ on the overall cross-species transmission dynamics of retroviruses. This implies that $\Omega$ is highest when $X y$ and $H s$ are closest, xeno-transplantations and culinary habits being better promoters of retroviral transmissibility than mere side by side contact. In situations of first retrovirus attempt on Hs, it should be noted that only Xy will have a field, the field around Hs being absent (, unlike the image is presented in Figure 3). Concealed but obviously inherent in the parabolic nature of $\Omega$ is the 'dual wave-particle' 
pattern of the imaginary retrovirological fields discussed above (see Figure 3 for a detailed illustration). In the light of further discussions (below) about the interactions between the retrovirological constant and the "retrovirological window", the retrovirological constant can itself be observed to demarcate the only true realms in which retrovirus cross-species transmission dynamics are predictably influenced by physical transmission.

\section{On the need for and nature of the retrovirological window $(\psi)$}

As observed in the Methods and Approach section (Annotation of the non-Euclidian biological tensors/scalars), what were arbitrarily annotated as physical tensors and scalars do not represent biological phenomena realistically, since such phenomena are simply innate or acquired ecological responses of the retrovirus/host to variations in population wide dynamics, and are probably subject to adaptive alterations. This led me to define the true realms of space-time within retrovirological fields in which the equation of retrovirology can be said to be most predictive of retrovirus cross-species transmission dynamics. To do so, I introduced the concept of a retrovirological window $(\boldsymbol{\psi})$. If the retrovirological constant $\boldsymbol{\Omega}$, as suggested above, may be defined graphically as the boundary of positivity in which the equation of retrovirology best predicts real events of retrovirus cross-species transmission dynamics that are most closely analogous to physical (non-biological) phenomena, then the retrovirological window $\psi$ is the mirror image of $\boldsymbol{\Omega}$ (see Figure 3 for a geometrical illustration of relationship between $\Omega$ and $\psi$ ). If $\psi$ is simply a mirror image completing the 2-D representation of what are actually 4-D psi-formalisms of $\Omega$, it is possibly not required in the equation. However, to account for its invisible influence on the overall dynamics of retroviral cross-species transmission, $\psi$ may be integrated by rephrasing the equation to $\psi \sqrt{ }|\mathbf{R T I}|$ $=\mathrm{Vm}{ }^{*} \mathrm{Ha}^{*} \Sigma \mathrm{Tt}{ }^{*} \Sigma \mathrm{As}^{*} \boldsymbol{\Omega}$ or $|\mathbf{R T I}|=\left(\mathrm{Vm}^{*} \mathrm{Ha}^{*} \Sigma \mathrm{Tt}{ }^{*} \Sigma \mathrm{As} \mathrm{s}^{*} \boldsymbol{\Omega}\right)^{\psi}$ so that, in 2-D visualization (where $\psi=2$ ), each tensor or scalar $\mathrm{Vm}, \mathrm{Ha}, \Sigma \mathrm{Tt}, \Sigma \mathrm{As}$ and $\Omega$ always has both a positive and negative true value.

This approach is borrowed from quantum mechanics, where the linear representation of the path of an electron or photon is represented mathematically by squaring the amplitudes to yield pulses in 2-D (see Figure 3) [54]. This also implies that, on a scale of $\mathrm{X} y$ to $\mathrm{H} s$, the integral of each of the normalized tensor and scalar functions $\mathrm{Vm}, \mathrm{Ha}, \Sigma \mathrm{Tt}, \Sigma \mathrm{As}$ and $\Omega$ is always one. Using the alternate approach that assumes dependency of Tt and AS within the same host, $|\mathbf{R T I}|=\left\{\boldsymbol{\Sigma}(\mathrm{Tt}-\mathrm{As})_{\mathrm{Xy}}{ }^{*} \boldsymbol{\Sigma}(\mathrm{As}-\mathrm{Tt})_{\mathrm{Hs}}\right.$ * $\left.\mathrm{Vm}^{*} \mathrm{Ha}^{*} \boldsymbol{\Omega}\right\}^{2}$.

\section{On the nature of space-time within biological systems}

Despite what may seem an apparent success in using non-Euclidian geometry to derive equations of retrovirology, several questions remain unanswered. First, if space-time in physics is four dimensional [43-46], is it appropriate to hold the same for biological phenomena, or are adjustments needed? The significance of this question is that, in Euclidean geometry, each tensor or scalar for (a) 2-D space-time has three components, (b) 3-D has six components, and (c) 4-D has 10 independent components [46]. In the non-Euclidean geometry that Einstein adopted for spacetime when deriving his field equations of gravitation (general relativity), each tensor had 16 components [43-45]. The question therefore becomes rational because, given 
that non-Euclidean geometry was borrowed to arrive at a general theory for the origins of retroviruses/retrovirology (meaning, we assumed biological space-time to be $4-\mathrm{D})$, the possible number of space-time dimensions in biology and the number of its components are open to inquiry.

Second, regardless of its finite composition, are the determinants of space-time in retrovirology limited to $\mathbf{V m}, \mathbf{H a}, \mathbf{T t}$, As, and $\boldsymbol{\Omega}$ or there more?

Third, are events in retrovirological space-time best regarded as particles, waves or dual? As shown in Figure 3, I have been led to adopt a 'dual wave-particle' representation of retroviral cross-species transmission dynamics [54].

These and possibly other issues that remain unclear leave the close-to-real physicomathematical representation of biology a matter for further inquiry.

\section{Hints on testing the equation of the theory of retrovirology}

Several unabridged gaps in experimental retrovirology are predicted by this unifying "equation of retrovirology", but many may be elucidated experimentally, underlining the need for further experimentation on the pathway of retroviral cross-species transmission to make the equation practically useful.

(1) Although data on the requirement for zoonotic viral mutations to achieve infection of humans are scanty, further experimental evidence is necessary to affirm the influence of $\mathrm{Vm}$ and $\mathrm{Ha}$ on the overall dynamics of retroviral cross-species transmission.

(2) The scope of both accepting scalars and transmissive tensors remains rather ambiguous and must be clarified. In addition, the correlation of changes in the individual components of the scalar and tensors with retroviral transmission dynamics must be corroborated by in-situ or in-vivo experimentation.

(3) Innovative techniques for the experimental quantification of components of both scalars and tensors affecting retroviral cross-species transmission are needed to define the finite measure of the retrovirological constant $(\Omega)$, even before we contemplate what its components are or may be. Perhaps field retrovirology may benefit from the following insights and propositions.

\section{(a) Experimental evidence for the requirement of $\mathrm{Vm}$ and $\mathrm{Ha}$ in retroviral cross-species} transmission

Completion of sequencing of several organismal genomes along with technological advances such as computation, software and web-based repositories of omes make it possible to obtain data not just on various mammalian and retroviral species genomes, but on their proteomes, transcriptomes, metabolomes etc. [55]. Although they are not yet appropriately unified to support retroviral work, such repositories, based on the entire omes of the virus and hosts before and after the establishment of competent retroviral cross-species transmission, can enable in-silico comparisons of viral and host omes to be made on either side. For instance, the retrovirus resource at NCBI's resource center for retroviruses (available at http://www.ncbi.nlm.nih.gov/retroviruses/) forms a useful starting point for constructing such virtual databases. Data obtained in these sorts of bioinformatics experiments will inform whether retroviral mutations such as those seen with pandemic influenza of swine or avian origin are always required to achieve 
retroviral transmission. In the same way it will enlighten us of the need for host adaptability at the molecular level (e.g. immunological), no matter how small.

\section{(b) Alternative in-silico, in-vitro, or in-situ experiments to derive evidence in support of} the role of variations within individual components of the accepting scalars and transmitting tensors in reservoir and new host

Affirmation of the relationship between the frequency of genomic integration hot spots (gIHS) and the rate of retroviral genome integration may be aided by using 3-D-based bioinformatics searches of 3-D host genomes or transcriptomes other than primary structure-based analysis[56,57]. Data from several mammalian genomes support the possible conservation of some candidate retrovirus integration hot spots such as LINE elements, Alu, CGp transcriptional sites and topoisomerase cleavage sites. However, in the light of uncertainty in existing data [25-33], it is necessary to determine experimentally whether retroviral gIHS are similar for all retroviruses in all hosts or whether they differ from one retrovirus or host to another, as current evidence suggests. Real time expression profiles of various virus-specific immunity (either by targeted Ellispot or proteome-wide association studies, PWAS) and evolutionary defenses such as RNAi (say, by transcriptome-wide association studies, TWAS), when correlated with the probability of viral integration and appropriately controlled for, may elucidate both the direction and the magnitude of their effect on retroviral cross-species transmission. $\mathbf{V m}$ may be measured as the ratio $\lambda^{\prime} / \lambda^{\mathbf{0}}$ and $\mathbf{H m}$ as the ratio $\phi^{0} / \phi^{\prime}$, finite values of $\lambda$ and $\phi$ being measured by automated sequencing and denoted as the number of unnatural base variations in the retrovirus and the host genome size in nucleotides.

\section{Conclusions}

Once such suggested and appropriately standardized experiments and techniques for the quantitative and qualitative determination of all SB tensors and scalars have been conducted, then, using real time data obtained from sampling of molecular epidemiology cohorts such as those recently described by Vandewoude et al. [9], one may not only test the theory advanced, but derive the finite equivalents of the retrovirological constant $(\boldsymbol{\Omega})$.

The practical value of the mathematical formalism proposed in this paper can then be assessed. This should include, I suggest, an inquiry into whether (1) the same model of inter-species transmission of infectious agents (zoonotic origins) may be extended without modification to the inter-species dynamics of other infectious agents, and (2) intra-species transmission dynamics of all human infectious agents can be predicted by a modified version of that model.

\footnotetext{
Acknowledgements

I thank Dr Paul Agutter for refining my ocean of words to drops of meaning. The relevance of peer review been evident as nowhere else, prompting me to thank the anonymous reviewers. Many persons have acted over a span of 10 years as sounding boards for these ideas, but the most influential were: Dr(s) Dhatemwa A. Muzaale, Johns Hopkins University, and Henry Kajumbula, Makerere University. At the latter, Prof(s) Joseph Olobo, Deo KadduMulindwa and Wilson Byarugaba have lately collaborated with me on actualizing some aspects of the same. No specific funding was received for this work.
}

Authors' contributions

WM conceived the hypothesis behind this work, designed and undertook the synthesis and derived the deductions WM also wrote the final draft of the manuscript. 


\section{Competing interests}

The authors declare that he has no competing interests.

Received: 12 October 2009

Accepted: 16 February 2010 Published: 16 February 2010

\section{References}

1. Ka S, Kerje S, Bornold L, Liljegren U, Siegel PB, Andersson L, Hallböök F: Proviral integrations and expression of endogenous avian leucosis virus during long term selection for high and low body weight in two chicken lines. Retrovirology 2009, 6:68.

2. Rauscher FJ, Allen BV: Growth curve of a murine leukemia virus in mice. J Nat/ Cancer Inst 1964, 32:269-275.

3. Wolfe ND, Switzer WM, Carr JK, Bhullar VB, Shanmugam V, Tamoufe U, Prosser AT, Torimiro JN, Wright A, MpoudiNgole E, McCutchan FE, Birx DL, Folks TM, Burke DS, Heneine W: Naturally acquired simian retrovirus infections in central African hunters. Lancet 2004, 363:932-937.

4. Gualco G, Chioato L, Weiss LM, Harrington WJ Jr, Bacchi CE: Analysis of human T-cell lymphotropic virus in CD25+ anaplastic large cell lymphoma in children. Am J Clin Pathol 2009, 132(1):28-33.

5. Hahn BH, Shaw GM, De Cock KM, Sharp PM: AIDS as a zoonosis: scientific and public health implications. Science 2000, 287:607-614.

6. Freed EO: HIV-1 replication. Somat Cell Mol Genet 2001, 26(1-6):13-33.

7. Brooks JI, Rud EW, Pilon RG, Smith JM, Switzer WM, Sandstrom PA: Cross-species retroviral transmission from macaques to human beings. Lancet 2002, 360:387-388.

8. Franklin SP, Troyer L, Terwee JA, Lyren LM, Boyce WM, Riley SP, Roelke ME, Crooks KR, Vandewoude S: Frequent transmission of immunodeficiency viruses among bobcats and pumas. J Virol 2007, 81(20):10961-10969.

9. Vandewoude S, Troyer J, Poss M: Restrictions to cross-species transmission of lentiviral infection gleaned from studies of FIV. Vet Immunol Immunopathol 2009, PMID 19896218.

10. Wayengera M: Emergence of zoonotic retroviral infections in this era. Why?. Abstract book and programme of Proceedings of the International Students' Conference on Emergencies (ISCE). 2001, Dec 11-14th 7.

11. Wayengera M: The evolutionary adaptation hypothesis to explain the origin of ebola VHF. Makerere Medical Journal 2002, 36:36-37

12. Heneine W: Emergence of novel retroviruses. Retrovirology 2009, 6(Suppl 2):15.

13. Bagasra O, Amjad M: Protection Against Retroviruses Are Owing to a Different Form of Immunity: An RNA-Based Molecular Immunity Hypothesis. Applied Immunohistochemistry \& Molecular Morphology 2000, 8(2):133-146.

14. Sopper S, Sauer U, Hemm S, Demuth M, Müller J, Stahl-Hennig C, Hunsmann G, Meulen V, Dörries R: Protective Role of the Virus-Specific Immune Response for Development of Severe Neurologic Signs in Simian Immunodeficiency Virus-Infected Macaques. J Virol 1998, 72(12):9940-9947.

15. Evans D, Bricker J, Lifson J, Lang S, Desrosiers R: Virus-specific Immune Responses in Macaques Inoculated with Single-cycle SIV. Conf Retrovir Oppor Infect-Boston Mass 2003, 10, abstract no. $78 .$.

16. Malim MH: APOBEC proteins and intrinsic resistance to HIV-1 infection. Phil Trans R Soc B 2009, 364(1517):675-687.

17. Okeoma CM, Low A, Bailis W, Fan HY, Peterlin BM, Ross SR: Induction of APOBEC3 In Vivo Causes Increased Restriction of Retrovirus Infection. J Virol 2009, 83(8):3486-3495.

18. Chiu Y-L, Greene WC: APOBEC3G: an intracellular centurion. Phil Trans R Soc B 2009, 364(1517):689-703.

19. Conticello SG, Thomas CJF, Petersen-Mahrt SK, Neuberger MS: Evolution of the AID/APOBEC Family of Polynucleotide (Deoxy)cytidine Deaminases. Molecular Biology and Evolution 2005, 22(2):367-377.

20. Kajaste-Rudnitski A, Pultrone C, Marzetta F, Ghezzii S, Coradin T, Vicenzi E: Restriction factors of retroviral replication: the example of Tripartite Motif (TRIM) protein 5alpha and 22. Amino Acids 2009, PMID: 19943174.

21. Tokarev A, Skasko M, Fitzpatrik K, Guatelli J: Antiviral Activity of the interferon induced cellular protein BST-2/ tetherin. AIDS Res Human Restroviruses 2009, PMID: 19929170.

22. Bagasra O, Prilliman KR: RNA interference: the molecular immune system. J Mol Histo 2004, 35(6):545-553.

23. Wayengera M: Pre-Integration gene slicing as an alternate or complimentary gene therapy modem to RNA interference. J Appl Biol Sci 2008, 1(2):54-63.

24. Hannon GJ, Rossi JJ: Unlocking the potential of the human genome with RNA interference. Nature 2004 431:371-378.

25. Vijaya S, Steffan DL, Robinson HL: Acceptor sites for retroviral integrations map near DNase I-hypersensitive sites in chromatin. J Virol 1986, 60:683-692.

26. Withers-Ward ES, Kitamura Y, Barnes JP, Coffin JM: Distribution of targets for avian retrovirus DNA integration in vivo. Genes Dev 1994, 8:1473-1487.

27. Sels FT, Langer S, Schulz AS, Silver J, Sitbon M, Friedrich RW: Friend murine leukaemia virus is integrated at a common site in most primary spleen tumours of erythroleukaemic animals. Oncogene 1992, 7:643-652.

28. Stevens SW, Griffith JD: Human immunodeficiency virus type 1 may preferentially integrate into chromatin occupied by L1Hs repetitive elements. Proc Natl Acad Sci USA 1994, 91:5557-5561.

29. Stevens SW, Griffith JD: Sequence analysis of the human DNA flanking sites of human immunodeficiency virus type 1 integration. J Virol 1996, 70:6459-6462

30. Howard MT, Griffith JD: A cluster of strong topoisomerase II cleavage sites is located near an integrated human immunodeficiency virus. J Mol Biol 1993, 232:1060-1068.

31. Bushman FD, Craigie R: Integration of human immunodeficiency virus DNA: adduct interference analysis of required DNA sites. Proc Natl Acad Sci USA 1992, 89:3458-3462.

32. Carteau S, Hoffmann C, Bushman F: Chromosome structure and human immunodeficiency virus type 1 cDNA integration: centromeric alphoid repeats are a disfavored target. J Virol 1998, 72(5):4005-4014.

33. Moalic $Y$, Blanchard $Y$, Félix $H$, Jestin A: Porcine endogenous retrovirus integration sites in the human genome: features in common with those of murine leukemia virus. J Virol 2006, 80(22):10980-10988. 
34. Tang J, Wilson CM, Schaen M, Myracle A, Douglas SD, Kaslow RA, REACH Study Group: CCR2 and CCR5 genotypes in HIV type 1-infected adolescents: limited contributions to variability in plasma HIV type 1 RNA concentration in the absence of antiretroviral therapy. AIDS Res Hum Retroviruses 2002, 18(6):403-412.

35. An P, Nelson GW, Wang L, Donfield S, Goedert JJ, Phair J, Vlahov D, Buchbinder S, Farrar WL, Modi W, O'Brien SJ, Winkler CA: Modulating influence on HIV/AIDS by interacting RANTES gene variants. Proc Natl Acad Sci USA 2002, 99(15):10002-10007.

36. Martin MP, Dean M, Smith MW, Winkler C, Gerrard B, Michael NL, Lee B, Doms RW, Margolick J, Buchbinder S, Goedert JJ, O'Brien TR, Hilgartner MW, Vlahov D, O'Brien SJ, Carrington M: Genetic acceleration of AIDS progression by a genetic variant of CCR5. Science 1998, 282:1907-1911.

37. Stephens JC, Reich DE, Goldstein DB, Shin HD, Smith MW, Carrington M, Winkler C, Huttley GA, Allikmets R, Schriml L, Gerrard B, Malasky M, Ramos MD, Morlot S, Tzetis M, Oddoux C, di Giovine FS, Nasioulas G, Chandler D, Aseev M, Hanson M, Kalaydjieva L, Glavac D, Gasparini P, Kanavakis E, Claustres M, Kambouris M, Ostrer H, Duff G, Baranov V, Sibul H, Metspalu A, Goldman D, Martin N, Duffy D, Schmidtke J, Estivill X, O'Brien SJ: Dating the origin of CCR5 delta 32 AIDS resistance gene by the coalescence of Haplotypes. Am J Hum Genet 1998, 62:1507-1515.

38. Faure S, Meyer L, Costagliolia D, Vaneensberghe C, Genin E, Autran B, Delfraissy JF, McDermott DH, Murphy PM, Debré P, Théodorou I, Combadière C: Rapid Progression to AIDS in HIV+ve individuals with a structural variant of the chemokine receptor CXC3CR1. Science 2000, 287:2272-2277.

39. Gonzales E, Kulkarni H, Bolivar H, Mangano A, Sanchez R, Catano G, Nibbs RJ, Freedman Bl, Quinones MP, Bamshad MJ, Murthy KK, Rovin BH, Bradley W, Clark RA, Anderson SA, O'Connell RJ, Agan BK, Ahuja SS, Bologna R, Sen L, Dolan MJ, Ahuja SK: The influence of CCL3L1 gene containing segmental duplications on HIV-1/AIDS susceptibility. Science 2005, 307:1434-1440

40. Beignon AS, McKenna K, Skoberne M, Manches O, DaSilva I, Kavanagh DG, Larsson M, Gorelick RJ, Lifson JD, Bhardwaj N: Endocytosis of HIV-1 activates plasmacytoid dendritic cells via Toll-like receptor-viral RNA interactions. J Clin Invest 2005, 115(11):3265-3275.

41. Miyauchi K, Kim Y, Latinovic O, Morozov V, Melikyan GB: HIV Enters Cells via Endocytosis and Dynamin-Dependent Fusion with Endosomes. Cell 2009, 137(3):433-444.

42. Pelchen-Matthews A, Raposo G, Marsh M: Endosomes, exosomes and Trojan viruses. Trends in Microbiology 2004, 4(7):310-316

43. Einstein A: Die Feldgleichungen der Gravitation. Sitzungsberichte der Preussischen Akademie der Wissenschaften zu Berlin 1915, 844-847.

44. Einstein A: Die Grundlage der allgemeinen Relativitätstheorie. Annalen der Physik 1916, 49.

45. Einstein A: Kosmologische Betrachtungen zur allgemeinen. Sitzungsberichte der Preußischen Akademie der Wissenschaften 1917, 142

46. Ricci T, Levi-Civita T: Méthodes de calcul différentiel absolu et leurs applications. Mathematische Annalen (Springer) 1900, 54(1-2):125-201.

47. Wayengera M: Slow Progression of paediatric HIV disease: Selective adaptation or chance phenomenon. Port Harcourt Medical Journal 2007, 2:83-87.

48. Smieszek T, Fiebig L, Scholz RW: Models of epidemics: when contact repetition and clustering should be included. Theor Biol Med Model 2009, 6:11.

49. Keeling MJ, Eames KTD: Networks and epidemic models. J R Soc Interface 2005, 2:295-307.

50. Wearing HJ, Rohani P, Keeling MJ: Appropriate models for the management of infectious diseases. PLoS Med 2005, 2: e174.

51. Read JM, Eames KTD, Edmunds WJ: Dynamic social networks and the implications for the spread of infectious disease. J R Soc Interface 2008, 5:1001-1007.

52. Eames KTD: Modelling disease spread through random and regular contacts in clustered populations. Theor Popul Biol 2008, 73:104-111.

53. Ferrari MJ, Bjornstad ON, Dobson AP: Estimation and inference of RO of an infectious pathogen by a removal method. Math Biosci 2005, 198:14-26.

54. Feynman RP: The Space-Time Formulation of Nonrelativistic Quantum Mechanics. Reviews of Modern Physics 1948, 20:367-387.

55. Wayengera $\mathrm{M}$, Byarugaba W: Emphasizing the vitality of genomics related research in the area of infectious diseases. Sci Res Essay 2008, 3(4):125-131.

56. Wayengera M, Byarugaba W, Kajumbula H, Olobo J, Kaddu-Mulindwa D: A model for mapping of Ebola and Marburg RNA integration sites in rhesus Macaca mulatta genome in silico: Ebola virus acceptors sites located on chromosomes 4, 6, 7, 8, 9, 14 and 15. Afr J Biotechnol 2009, 8(10):2125-2130.

57. Wayengera M, Kajumbula H, Kaddu-Mulindwa D, Olobo J, Byarugaba W: Proteomics of Marburg and Ebola glycoproteins: Insights into their physicochemical similarities and irregularities. Afr J Biotechnol 2009, 8(17):4025-4031.

58. Biology of HIV. [http://www.web-books.com/eLibrary/ON/B0/B22/05MHIV.html].

doi:10.1186/1742-4682-7-5

Cite this article as: Wayengera: On the general theory of the origins of retroviruses. Theoretical Biology and Medical Modelling 2010 7:5. 